\title{
Radio evidence for breakout reconnection in solar eruptive events
}

\author{
H. Aurass ${ }^{1}$, G. Holman ${ }^{2}$, S. Braune ${ }^{1}$, G. Mann ${ }^{1}$, and P. Zlobec ${ }^{3}$ \\ ${ }^{1}$ Leibniz-Institut für Astrophysik Potsdam (AIP), 14482 Potsdam, Germany \\ e-mail: haurass@aip.de \\ 2 NASA Goddard Space Flight Center, Code 671, Greenbelt, MD 20771, USA \\ 3 INAF - Trieste Astronomical Observatory (OAT), via G.B. Tiepolo 11, 34143 Trieste, Italy
}

Received 15 January 2013 / Accepted 21 May 2013

\begin{abstract}
Context. Magnetic reconnection is understood to be fundamental to energy release in solar eruptive events (SEEs). In these events reconnection produces a magnetic flux rope above an arcade of hot flare loops. Breakout reconnection, a secondary reconnection high in the corona between this flux rope and the overlying magnetic field, has been hypothesized. Direct observational evidence for breakout reconnection has been elusive, however.

Aims. The aim of this study is to establish a plausible interpretation of the combined radio and hard X-ray (HXR) emissions observed during the impulsive phase of the near-limb X3.9-class SEE on 2003 November 03.

Methods. We study radio spectra (AIP), simultaneous radio images (Nançay Multi-frequency Radio Heliograph, NRH), and singlefrequency polarimeter data (OAT). The radio emission is nonthermal plasma radiation with a complex structure in frequency and time. Emphasis is on the time interval when the HXR flare loop height was observed by the Ramaty High Energy Solar Spectroscopic Imager (RHESSI) to be at its minimum and an X-ray source was observed above the top of the arcade loops.

Results. Two stationary, meter-wavelength sources are observed radially aligned at 0.18 and $0.41 R_{\odot}$ above the active region and HXR sources. The lower source is apparently associated with the upper reconnection jet of the flare current sheet (CS), and the upper source is apparently associated with breakout reconnection. Sources observed at lower radio frequencies surround the upper source at the expected locations of the breakout reconnection jets.

Conclusions. We believe the upper radio source is the most compelling evidence to date for the onset of breakout reconnection during a SEE. The height stationarity of the breakout sources and their dynamic radio spectrum discriminate them from propagating disturbances. Timing and location arguments reveal for the first time that both the earlier described above the flare loop top HXR source and the lower radio source are emission from the upper reconnection jet above the vertical flare CS.
\end{abstract}

Key words. Sun: radio radiation - Sun: X-rays, gamma rays - Sun: coronal mass ejections (CMEs) - Sun: magnetic topology Sun: flares - Sun: corona

\section{Introduction}

Solar eruptive events (SEEs), events displaying the combination of a flare and coronal mass ejection (CME), are due to coronal energy release with different characteristic temporal and spatial scales, but comparable total energy content (Holman 2012). In this paper we re-investigate a well-studied SEE, the 2003 November 03 X3.9 flare/CME event of interest (EOI). The flare occurred in NOAA AR10488 in the northern hemisphere, and near the west limb (at N07W72). It was the subject of a sequence of earlier papers (Dauphin et al. 2005; Vršnak et al. 2005, 2006; Dauphin et al. 2006; Veronig et al. 2006; Chen \& Petrosian 2012). Here we consider the impulsive phase of the event, concentrating on key details of high time resolution complementary radio data. For general information about radio flares see the review of Bastian et al. (1998).

The importance of the effects in radio emission presented here, and which at first glance seem to be minor, follows from their time synchronization with the dynamics of the hard $\mathrm{X}$-ray (HXR) flux and flare loop geometry determined in previous works. Henceforth, the frequently occurring reference to Veronig et al. (2006) is abbreviated as V06.

The well-known standard model for SEEs, such as described in Lin \& Forbes (2000); Lin et al. (2004), reflects many observational facts. The essence of the model is the loss of the equilibrium of a previously stable configuration of an arcade of magnetic loops, leading to the formation of a flux rope and a current sheet (CS) underneath, and a new, less-sheared arcade of loops below that.

Observations of the early stages of CS formation, and the proper onset of the eruptive energy release are rare if not altogether missing. They are, nevertheless, essential: they would encompass the transition from slow (heating) to fast (eruptive) energy release, from current dissipation due to normal resistivity to fast CS development thanks to anomalous resistivity in the current dissipation region. The transition from slow to fast development takes place on spatial and temporal scales of the dilute and hot coronal plasma which are small (meters) and short (subseconds), respectively.

We are investigating if we recognize this transition and the influenced regions of the coronal magnetic field by tracing the large-scale distribution of newly formed radio source sites around the flaring active region. Newly assembled and re-considered observations are presented, supporting the view sketched in Fig. 1. For limb events such as the EOI, radio observations as in Aurass et al. (2011) could give further evidence for the process of coronal breakout reconnection, as was initially suggested by Antiochos et al. (1999), and also discussed by, e.g., MacNeice et al. (2004) and Karpen et al. (2012). 


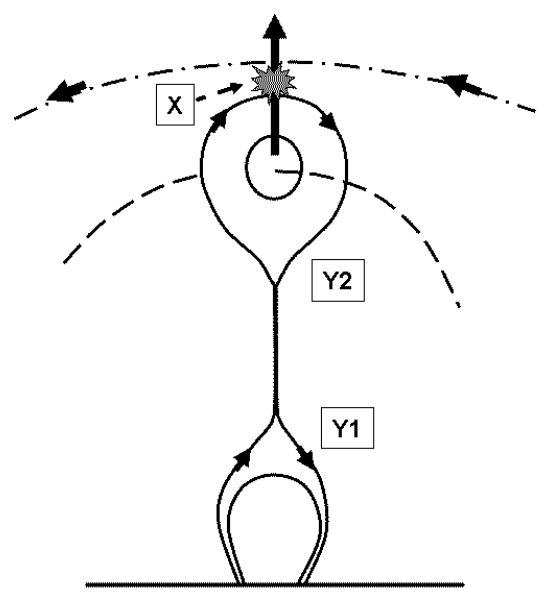

Fig. 1. Schematic of an erupting AR filament. Initially independent global flux systems (dash-dotted line) are at the top. Underneath the filament (flux rope) a CS is formed terminating in two Y-points with outflow jets. The interaction of the rising flux rope with the global field may drive X-point (breakout) reconnection. The dashed line indicates extension of this $2 \mathrm{D}$ slice into $3 \mathrm{D}$.

Using the setup in Fig. 1, we are attempting to locate specific radiation signatures related with the initiation of the hot, turbulent, and dense plasma structures around the magnetic branch points Y1, Y2, and X. After introducing the radio and HXR data, we describe the radio spectral and imaging data of special relevance for understanding the coronal breakout phenomenon in the EOI. We discuss the in-phase development of the radio and HXR signatures, and conclude with a discussion of the relevance of our results to the general understanding of SEEs.

\section{The radio and hard X-ray data: an overview}

The start of the flare impulsive phase is commonly defined by the rise of the HXR flux due to the onset of a fast release of magnetically stored energy in coronal volumes which are too small to be resolved by any existing imaging observations. The term impulsive describes the sudden rise of the flux over time. Sometimes there is evidence for a pre-heating phase, a gradual flux enhancement of thermal radio and soft X-ray emissions (e.g. Svestka 1976; Altyntsev et al. 2012).

The fast release of magnetic energy in the dilute coronal plasma is the reason why a huge fraction of energy is carried away by accelerated particles. The 10-30 keV electron component excites nonthermal meter and decimeter radio burst emission via plasma processes (Melrose 1985). Considered with the necessary time resolution, the occurrence of notable impulsive radio signatures may not coincide in time with the impulsive HXR emission. This is reasonable, because of the different excitation conditions of the radiations and the different source sites.

Theoretical considerations of eruptive instabilies in the corona (Spicer 1977) result in a prefered parameter range; passing through the corona radially outward, the magnetic field must still be large enough (as the energy source) and the density small enough to force the instability development. In the meter wave range, our observational experience points to the preflare $\sim 300 \mathrm{MHz}$ plasma frequency level in the active region corona where the very first nonthermal flare burst signatures occur. In Fig. 2a we present the contrast-enhanced dynamic radio spectrum of the corresponding time interval of the EOI.
The RHESSI data of the EOI are analyzed in V06. There are determined the parameters of the evolving coronal HXR loop above the footpoint sources in the low atmosphere, and described the quadrupolar structure of the flaring AR10488. In the EOI the HXR loop can be imaged nicely at the very beginning of the flare; the loop top is identified in the range 10-30 keV at 09:46 UT, and the footpoints in 70-100 keV at 09:48:50 UT. The loop top height shrinks to a minimum of $7 \mathrm{Mm}$ between 09:46 and 09:48:30 UT, and stops there until 09:49:20 UT. At 09:49:50 UT, the HXR loop top is already rising, and continues until the end of the RHESSI day. In Fig. $2 b$ we have arranged several special features of the HXR and radio data on the same time scale as Fig. 2a which will be discussed in the next sections. The boldfaced asterisks denote the data points of the HXR height-time plot as given in V06.

A secondary HXR source, the above the loop top source (Fig. 2b, and the inset in our Fig. 7) appears at 09:47:52 UT, culminates at $31 \mathrm{Mm}$ (projected westward distance from the active region) at 09:49:13 UT, and disappears from the 15-20 keV energy range after 09:49:29 UT (V06, their Fig. 9.). This phenomenon is known from other events (Sui et al. 2004; Liu et al. 2009; Holman et al. 2011), but is not yet fully understood (see also Chen \& Petrosian 2012).

We find that several well-defined radio features precisely follow the HXR timing, as they should if the HXR-exciting electrons are accelerated in the solar corona. The identification of the sites and the mechanisms of particle acceleration is a key problem of solar (and astro-)physics.

The data analysis will lead us to the occurrence and evolution of current sheets in models of coronal eruptive processes. Magnetic reconnection at coronal current sheets is probably the best accepted approach to a mechanism of fast transformation of magnetic into kinetic and thermal energy. For reference we quote here Priest \& Forbes (2000); Somov (2000) for general aspects, and, e.g., Mann \& Warmuth (2011) and Holman et al. (2011) for refinements, mainly based on RHESSI observations.

\section{Radio emission during the HXR loop depression}

Always keeping in mind Fig. 2, we consider the interval from the first HXR loop top visibility (09:46 UT) until the height minimum transit and the onset of its fast lift-up (09:49:20-50 UT). This must be the interval of the most efficient electron acceleration; the loop top HXR source achieves its emission ratio ${ }^{1}$ maximum.

The enlarged dynamic spectrum in Fig. 3a reveals that a morphological change occurs as the frequency increases from below $250 \mathrm{MHz}$ to above $280 \mathrm{MHz}$. With rising frequency the spectral features change from patchy and irregular with small characteristic bandwidth and duration, to fast drift bursts. These are sometimes similar to inverted-U bursts (e.g., Aurass \& Klein 1997) or to herringbone bursts (a fine structure of type II radio bursts, e.g., Mann \& Klassen 2005) with a characteristic knifeedge time profile.

With regard to this morphological boundary in the dynamic spectrum, we divide the radio image analysis into a range with fast drift features ( $\geq 327 \mathrm{MHz}$, Sect. 3.1), and a range at lower frequencies with patchy features (imaged at NRH 236.6 MHz, Sect. 3.3). Based on the high time resolution and sensitive OAT single frequency polarimeter records we find that faint and highly polarized subsecond pulsations between 450 and $300 \mathrm{MHz}$ occur just before the first strong fast drift feature

1 This is the count rate ratio $(20-25 \mathrm{keV}) /(15-20 \mathrm{keV}), \mathrm{V} 06$. 


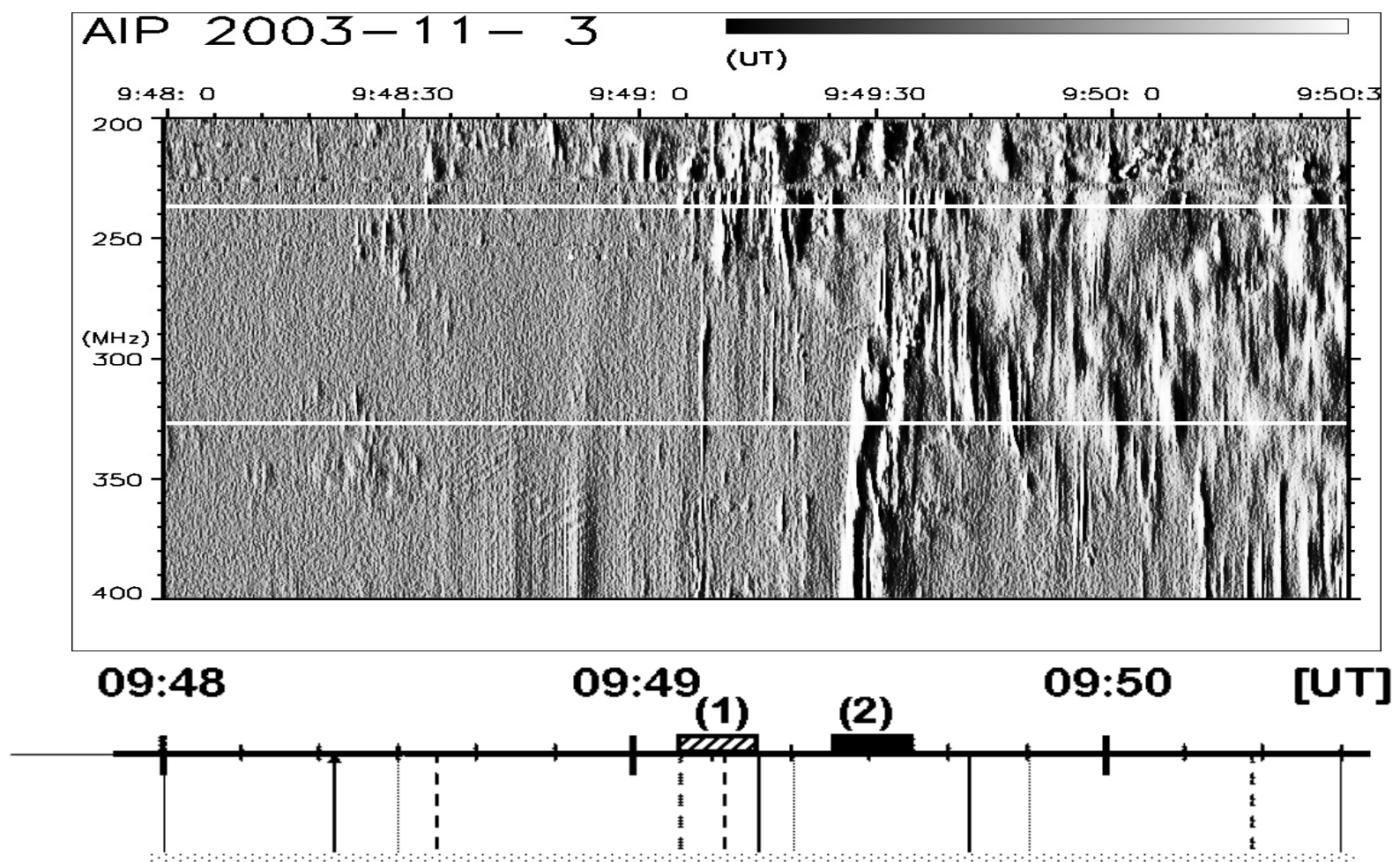

a)

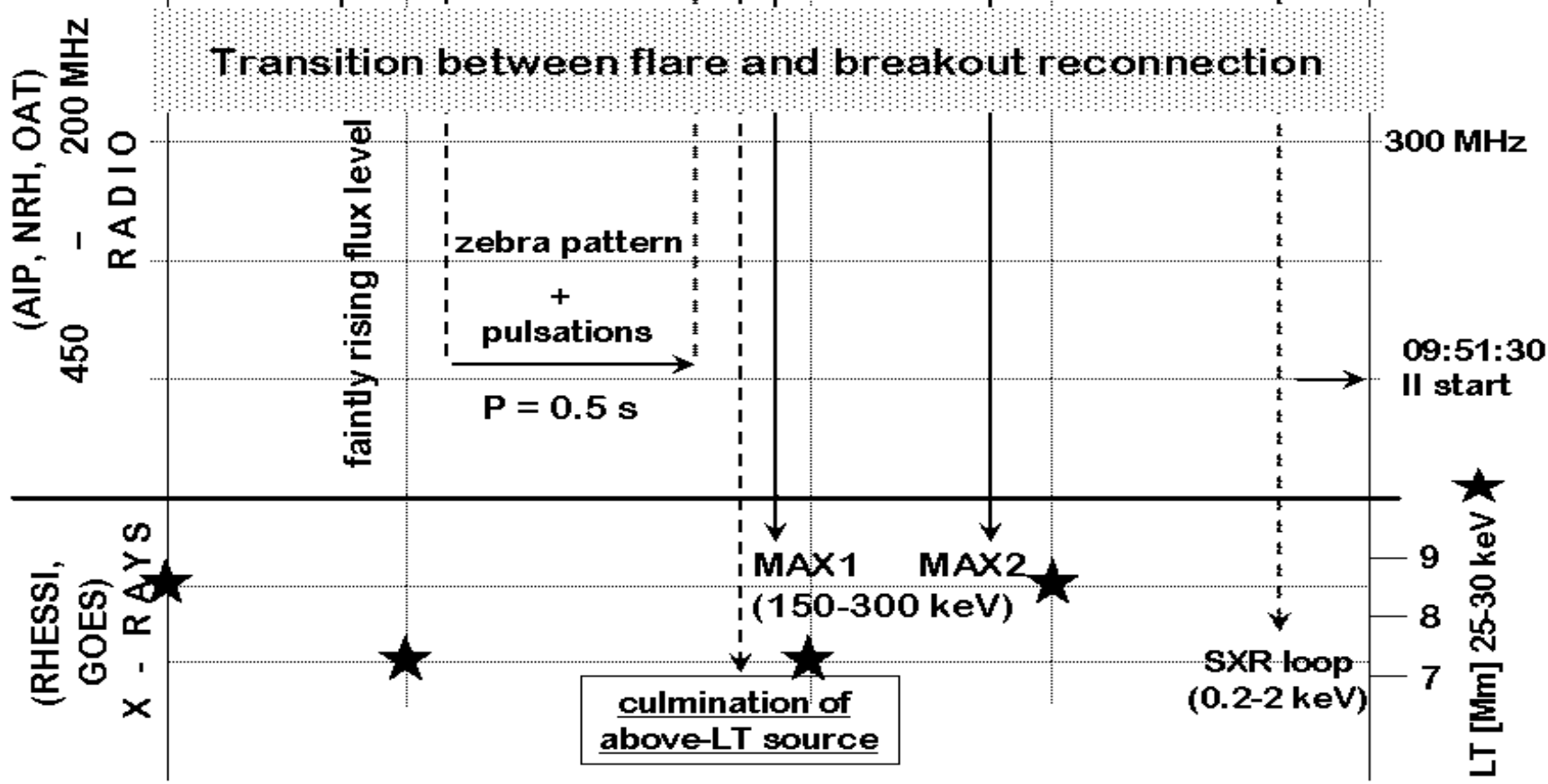

b)

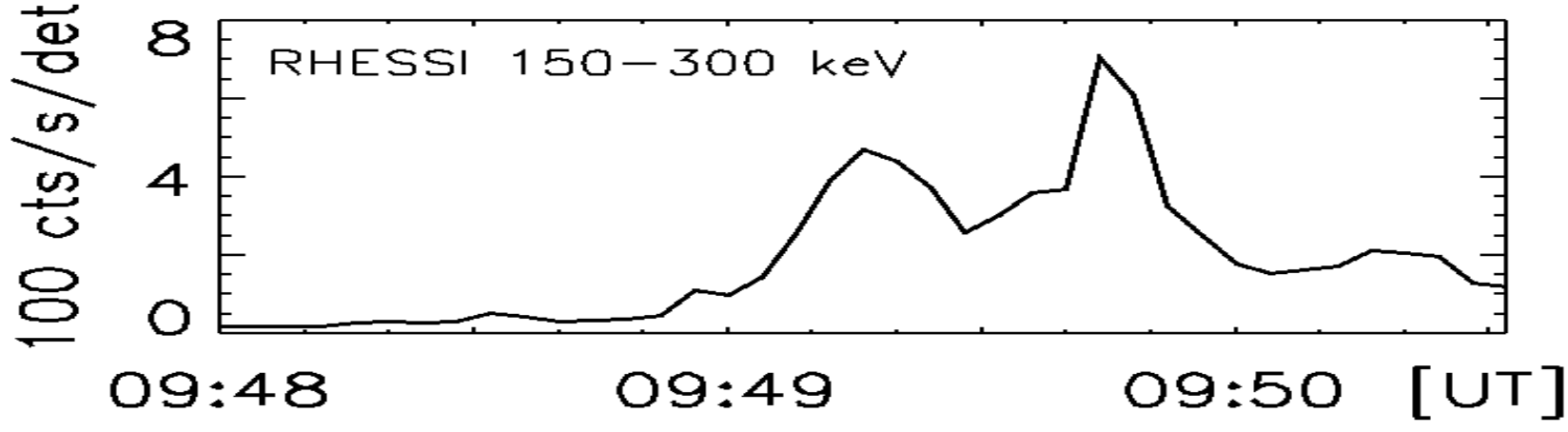

Fig. 2. 2003 November 03 impulsive phase synopsis. a) The AIP dynamic spectrum; NRH frequencies indicated by white lines. b) Radio and X-ray data time line. For the soft X-ray (SXR) loop see Dauphin et al. (2006). c) RHESSI HXR counts (this and the boldfaced asterisks in b) following V06). 

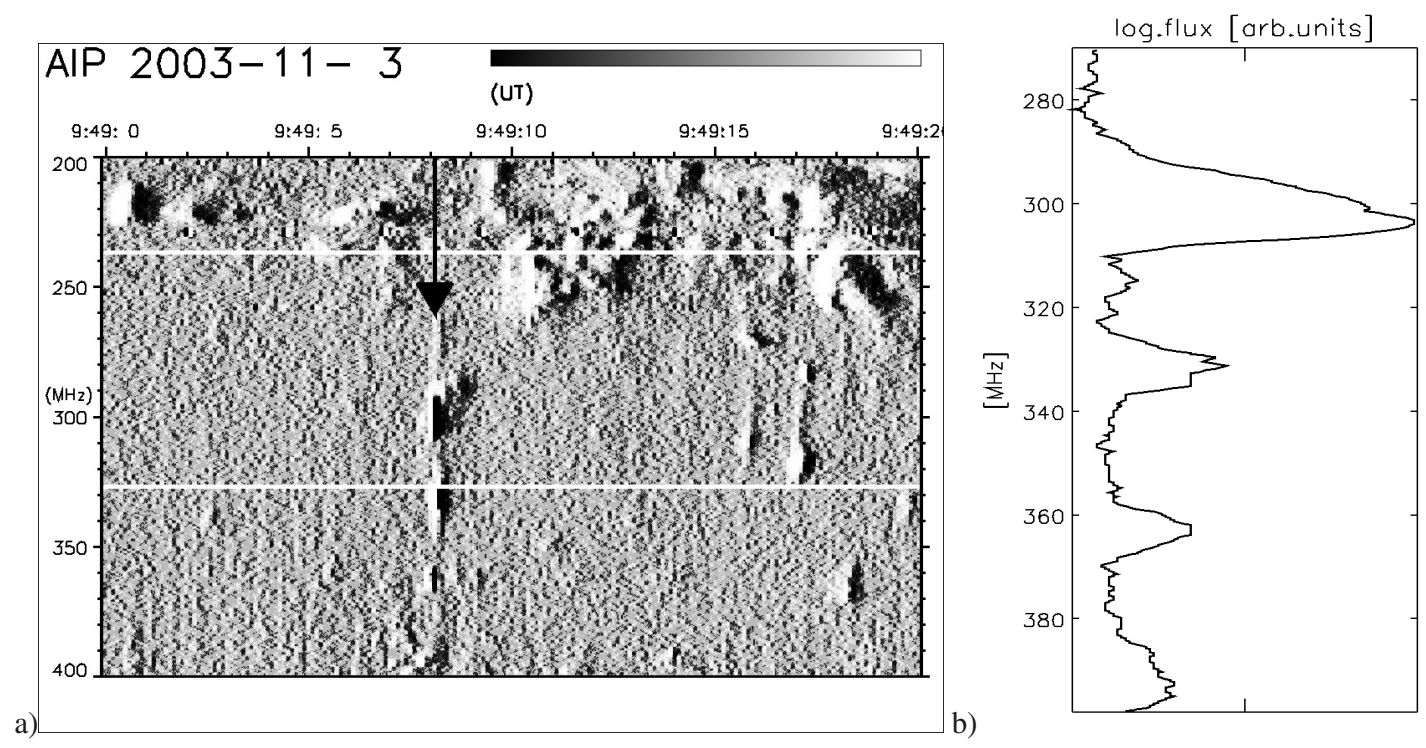

Fig. 3. Feature (1) of Fig. 2b. In the dynamic spectrum, the white lines denote the NRH frequencies. The black arrow points to the time of the instantaneous spectrum shown in b), on a $\log$ (flux) scale in relative units. The frequency scales in a) and b) are different.

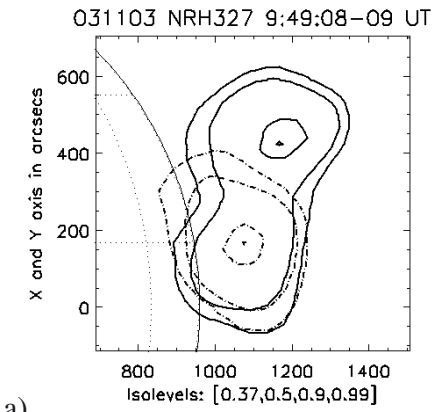

a)

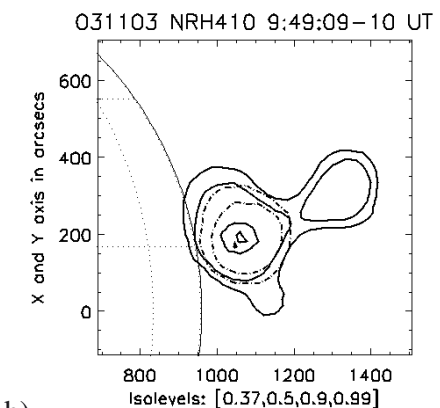

b)

Fig. 4. NRH-observed radio source evolution at a) $327 \mathrm{MHz}$, 09:49:08 (dash-dot) vs. 09:49:09 UT, and b) 410.5 MHz, 09:49:09 (dash-dot) vs. 09:49:10 UT. NRH $432 \mathrm{MHz}$ is similar and not shown here. Earlier, in the lower source a highly polarized component fluctuates with a period of $0.5 \mathrm{~s}$ (Sect. 3.2). All images are normalized together in $[0,1]$ before plotting.

(Sect. 3.2). In Sect. 3.4 we draw a composite figure of the X-ray and radio signatures of the beginning of the impusive flare phase. The continuing development until the bright radio type II burst shows signs of coronal relaxation where the coronal breakout radio source dominates all other source sites (Sect. 3.5).

\subsection{Frequencies $\geq 327 \mathrm{MHz}$}

The radio feature in the AIP spectrogram enlarged in Fig. 3a is, according to our best knowledge, the first report of a simultaneous radio phenomenon with the HXR above the loop top source (compare Fig. 2b). The feature has a duration of about $1 \mathrm{~s}$ and a highly structured spectrum. There is a spectral maximum at $303 \mathrm{MHz}$ and, with a persistent frequency shift of $30 \mathrm{MHz}$, there are at least three further peaks at higher frequencies (see the instantaneous spectrum in Fig. 3b, taken at the time indicated by the black arrow). Another, much stronger spectral feature starts $20 \mathrm{~s}$ later (Sect. 3.5).

Figure 4 demonstrates the dramatic radio source evolution seen in the NRH radio images between the seconds 09:49:08-09 (at $327 \mathrm{MHz}$, panel a) and 09:49:09-10 UT (at 410 and
$432 \mathrm{MHz}$, panel b). The state before the changes is plotted in dashed isolines; the new state is given in the continuous isolines. We note that the radio maps were calculated after averaging the uncompressed NRH data to $1 \mathrm{~s}$ time resolution. All maps shown here are normalized together so they express the relative importance of changes over time and frequency.

The radio images reveal how a previously very faint source site with a centroid at about $123 \mathrm{Mm}$ above the active region ${ }^{2}$ is suddenly accompanied by an additional source at a height of about $290 \mathrm{Mm}$, much higher up in the corona. The situation is almost the same at 410 and $432 \mathrm{MHz}$. At $327 \mathrm{MHz}$ the upper source is turned by a position angle of about $10 \mathrm{deg}$ northeast to the radial direction drawn from the center of the disk through the active region and the lower radio source centroid. Veronig et al. (2006) report a deviation of $18 \mathrm{deg}$ to the radial direction for the rise of the HXR loop-top source in the same direction.

We regard the lower radio source to be due to electron acceleration in the upper outflow jet (Y2 in Fig. 9) of the growing flare reconnection process, and the upper radio source to be the first radio light of breakout reconnection at the X-point between flare-related, rising flux systems, and global overlying magnetic flux systems (X in Fig. 9).

\subsection{Pulsations indicate the instability onset}

About $40 \mathrm{~s}$ before the first evidence of a radio source in the upper reconnection outflow jet, and of the radio brightening in the overdense plasma around the breakout reconnection X-point, the AIP dynamic spectra and the high time resolution OAT polarimeters reveal faint fluctuations in the frequency range $300-450 \mathrm{MHz}$ (Fig. 2). They are shown enlarged in Fig. 5.

The pulsations are best visible at the OAT $408 \mathrm{MHz}$ record; at OAT $327 \mathrm{MHz}$ the pulsations are mixed with a simultaneously occurring zebra pattern (Zlotnik et al. 2003 as reference of the radio spectral fine structures). In Fig. 5a the unprocessed observations are shown in both OAT $408 \mathrm{MHz}$ circular polarization channels (IR, IL). Fast fluctuations are only visible in IR, which means they are highly polarized. The receiver noise is

\footnotetext{
2 All heights are given in projection; the reference point is between the HXR footpoint sources in AR10488.
} 


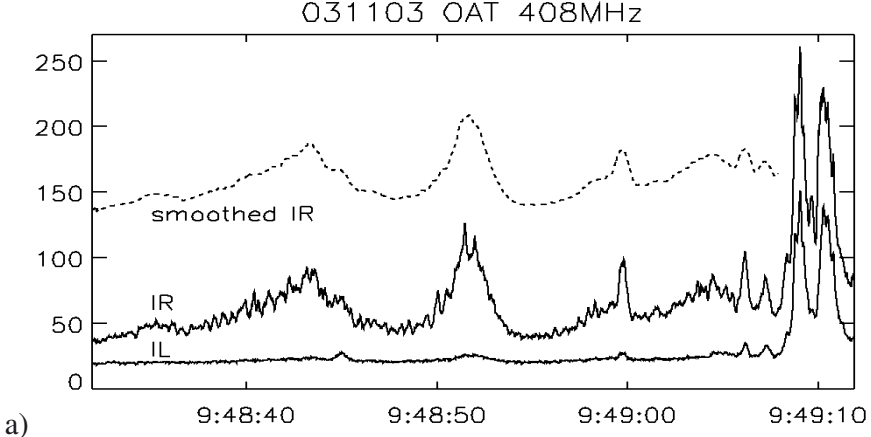

IR - smoothed IR

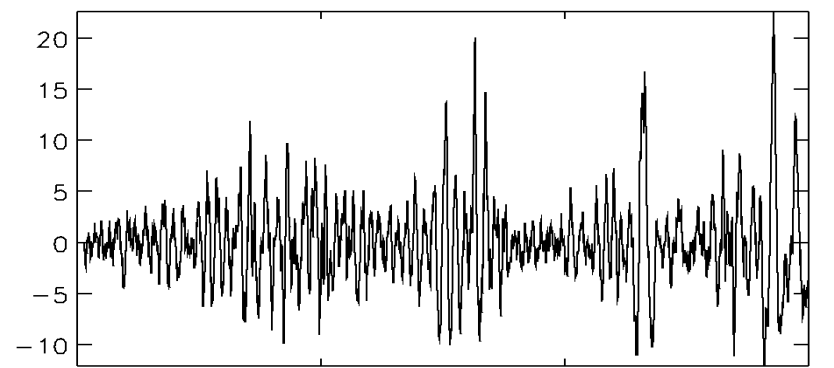

b) $9: 48: 30$

$9: 48: 42$

$9: 48: 54$

$9: 49: 06$

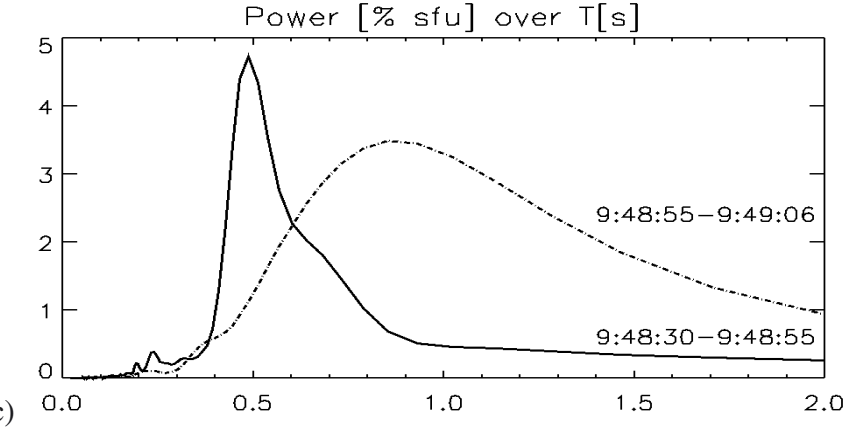

Fig. 5. OAT $408 \mathrm{MHz}$ data. a) IR and IL. The dashed curve (smoothed IR) is raised to avoid overlap. b) The fast pulsations in IR. We note that the time axes of a) and b) are different; the $y$-axis is in sfu $\left(1\right.$ s.f.u. $\left.=10^{-22} \mathrm{Ws} \mathrm{m}^{-2}\right)$. c) The power spectrum of two segments of b). The $0.5 \mathrm{~s}$ period is sharply defined.

far below the fluctuation amplitude (compare both channels in Fig. 5a). In the same panel, the dashed curve (raised by an arbitrary amount in flux for clarity) shows the IR component where the fast fluctuations were smoothed out. It remains a partly polarized component (visible in IR, and much fainter in IL, too) with a quasi-period of about $7 \mathrm{~s}$. The pulse sequence gets shorter with time. With the NRH 410.5 MHz uncompressed imaging data we qualitatively checked that the source above AR10488 is the only fluctuating source on the sun.

The described fast radio flux variations immediately precede in time the important spectral pattern of Fig. 3, indicating the injection of accelerated electrons. It seems that the electron acceleration is modulated with a quasi-period of $7 \mathrm{~s}$. This process is quite naturally accompanied by the rise of the radio (and HXR) flux. The simultaneous zebra-stripe excitation demands an electron population with a strong temperature anisotropy in the source volume (e.g., Zlotnik et al. 2003; Chen et al. 2011). The high polarization degree generally requires a compact source region and coherent radio emission.

We observe about 80 periods of the 0.5 s-oscillations, which are not damped, but vary in amplitude. The spectral peak obtained from the first 25- second segment of the record is a
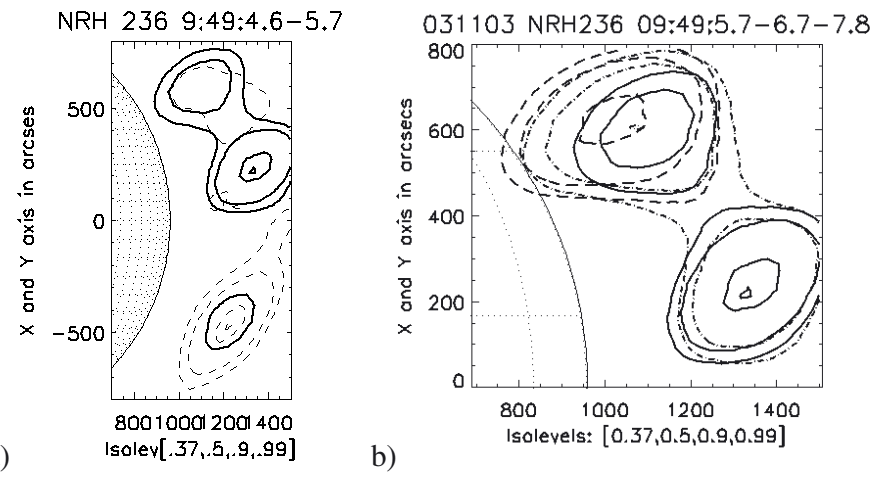

Fig. 6. NRH radio source evolution at 236.6 MHz: a) 09:49:04.6 (dashed) to 09:49:05.7 UT (continuous). The flux center of gravity jumps from AR10484 in the south to AR10488. b) 09:49:05.7 (cont.), 09:49:06.7 (dash-dot), 09:49:07.8 UT (dash). The images in b) are normalized in $[0,1]$.

convincing result (the continuous curve in Fig. 5c). The broader spectrum (the dashed curve) of the last 11 seconds of the interval may be due to the shorter data segment, and due to the irregular mixing with less polarized pulses that come from a site approaching the later upper source site.

Summarizing the behavior of the radio continuum fine structures in the 40 seconds before the radio and HXR evidence of the eruption onset, we conclude that they characterize the dynamics of the transition from the quasi-static preheating phase to the eruptive energy release. As already mentioned above, this phase transition is commonly associated with the changing electrical resistivity in the flare CS from normal to anomalous resistivity (Priest \& Forbes 2000; Somov 2000). We emphasize that this happens together with the arrival of the HXR loop top at its lowest height and turning point (Fig. 2b), given the accuracy and sampling of the loop-top height measurements of V06.

\subsection{Frequencies $<327 \mathrm{MHz}$}

Figure 2a shows diffuse patches of emission between 280 and $200 \mathrm{MHz}$, already present before the effects at higher frequencies. In the range $80-40 \mathrm{MHz}$ (not shown here) diffuse drift bursts occur of type III and/or type U after 09:48:54 UT.

In Fig. 6a, the radio source evolution is demonstrated at $236.6 \mathrm{MHz}$ just before the state shown in Fig. 4: at 09:49:04.7 UT, the emission above a southern active region (dashed) dominates. But one second later, at 09:49:05.7 UT, a stronger double source is formed above AR10488. This picture remains stable, with the tendency of a brightness shift farther and farther toward the northeastern source (Fig. 6b). There is no 236.6 MHz source at lower heights. We conclude that in the given event, the source location and structure at frequencies less than $250 \mathrm{MHz}$ are completely different from the higher frequencies. This is not really surprising, considering the different spectral features below and above the $250 \mathrm{MHz}$ range.

\subsection{Synthesis}

Figure 7 is an overlay of the different radiation signatures observed in radio (isolines, Sects. 3.1 and 3.3) and hard X-rays (the RHESSI-image after Fig. 9 of V06) in the corona above AR10488 during the time interval in Fig. 3. It is denoted as segment (1) on the time axis in Fig. 2 b. 


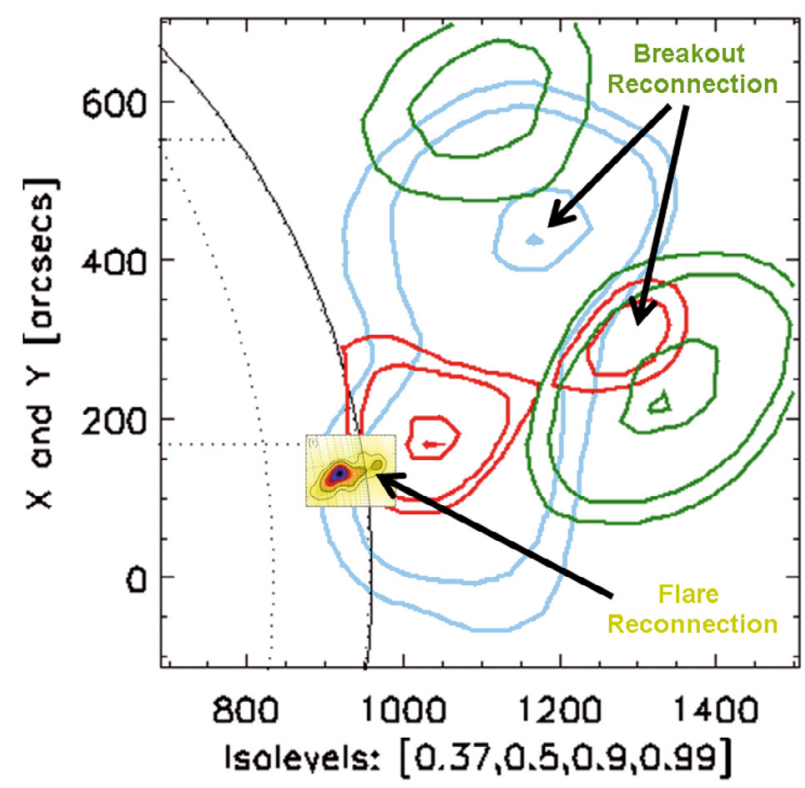

Fig. 7. Composed source site arrangement at the very beginning of the flare impulsive phase. NRH radio isolines: red: $410.5 \mathrm{MHz}$; cyan: $327 \mathrm{MHz}$; green: 236.6 MHz. The inset denoted as Flare Reconnection is the associated RHESSI-HXR image from V06's Fig. 9.

On the plane of the sky, the low-frequency sources (dark green isolines) flank the centroid of the upper high-frequency sources (upper red and light blue isolines), as is expected for a breakout reconnection CS (at point X of Fig. 1) with a perpendicular orientation to the flare CS (between Y2 and Y1 in Fig. 1). The flare CS is invisible in the frequency range of our radio observations. We associate the lower radio source (the lower part in red and light blue isolines in Fig. 7) with radio emission of the upper flare reconnection outflow jet. It seems that the above the flare loop top HXR source can be identified with the same flare reconnection outflow jet, but situated nearer to the point Y2 of the scheme in Fig. 1, and that the point Y1 and the lower flare reconnection outflow are immediately above the HXR flare loop top.

It seems as if the evolution at higher frequencies needs an opening of magnetic coronal structures which are closed before. This possibility is suggested by the motion of the northeastern source (dark green isolines in Fig. 7) and the fast decay of the southwestern source at 236.6 MHz. It is also supported by the onset delay of the meter wave flare-related phenomena: they are first observed at $236.6 \mathrm{MHz}$, later at higher frequencies. This resembles the magnetic breakout mechanism first proposed by Antiochos et al. (1999), and indicated in radio emission for a disk-event observed by Aurass et al. (2011).

\subsection{The following minutes}

As shown in Fig. 2a, there is a strong pulse of spectrally complex structured radio emission around 09:49:30 UT, labeled interval (2) at the top of Fig. 2b. It is the strongest meter-wave spectral pattern of the impulsive phase occurring before the HXR count rates grow to $>200 \mathrm{~s}^{-1}$ per detector (see Fig. 2c). Notice that this feature is not at all a type III burst as assumed by Chen \& Petrosian (2012). Several positively drifting features resemble the herringbone type II burst fine structure. Later (until 09:51:30 UT, the onset of the still brighter type II burst emission, not shown here) the characteristic spectral pattern of the range $280-330 \mathrm{MHz}$ expands to the range $250-\geq 400 \mathrm{MHz}$, but persists in a sequence of numerous narrowband fast drift bursts of both frequency drift rate signs.

The spatial source arrangement (Fig. 8b) is slightly different from Fig. 7. The upper sources coincide at $\geq 327 \mathrm{MHz}$ in the middle of their previous positions. The 236.6 $\mathrm{MHz}$ emission (not shown in Fig. 8b) forms a double source as before, with the northeastern source dominating. The densities are enhanced up to seven times compared with a $1.4 \mathrm{MK}$ barometric density model, as already found for feature (1) in Fig. 2.

Although the upper source site dominates the radio image, lower fainter sources are seen in Fig. 8b to the northeast and southwest of the active region. The $327 \mathrm{MHz}$ sources are located farther from the active region than the $410,5 \mathrm{MHz}$ sources, indicating lower densities away from the active region on both sides. These lower sources may originate from locations along the reconnected breakout field where the plasma density has increased downward from the reduced density region above to values appropriate for the radio emission.

It is important to note that in the entire time period from 09:49:08 until 09:51:30 UT (about 140 s) all the considered radio sources do not move radially farther outward than at 09:49:08 UT, and look very complex in the dynamic radio spectrum. This is the essential difference between the breakout reconnection sources on the one hand, and moving shock sources ahead of a blast wave, a flare ejection, or a CME on the other hand. Furthermore, we repeat that, in principle, in the radio emission nothing new is happening in the spectrum after the occurence of feature (1) in Fig. 2. The state remains stable until the onset of the flare blast wave radio emission of type II which starts several arcsecs farther out from the source centroid shown in Fig. 8b.

\section{Summary and conclusions}

We believe the presented observations provide the best evidence to date for breakout reconnection in a SEE. To simplify this summary we have re-plotted in Fig. 9 the schematic shown in Fig. 1 integrated with our findings; to the left the heights of the features are given as derived for the EOI.

In the range 327,410 , and $432 \mathrm{MHz}$ (the upper discrete $\mathrm{NRH}$ imaging frequencies), two sources are observed. One is radially above and slightly north of the other, at 09:49:08-09:49:10 UT, during the onset of the rise to the impulsive HXR peak of the flare and at the time when the centroid of the loop-top HXR source is at its lowest height. The centroid of the lower radio source is above the centroid of the above the loop top HXR source. This lower source is plausibly associated with electrons accelerated in the upper reconnection jet from the flare CS located between the loop-top and above the loop top HXR sources. The lineup of lower frequency radio sources on either side of the upper $410.5 \mathrm{MHz}$ source (here shown at NRH 236.6 MHz), along a curve roughly perpendicular to the line between the two $410 \mathrm{MHz}$ sources, indicates that these sources are associated with electron acceleration during breakout reconnection.

The observations reveal that the preflare configuration reached a regime where the previously radio-invisible flare and breakout CSs enter into a new phase. This transition is announced by the occurrence of the circularly polarized periodic flux oscillation of the pre-existing faint source (duration about $40 \mathrm{~s}$, period $0.5 \mathrm{~s}$ ) and the excitation of a zebra pattern. In the same time interval the HXR loop top in the flaring active region descends to a minimum height of about $7 \mathrm{Mm}$ (V06). A narrowband multi-lane radio feature in the spectrum in Fig. 3a, mapped as the above-mentioned pair of sources in Fig. 4, signifies the 

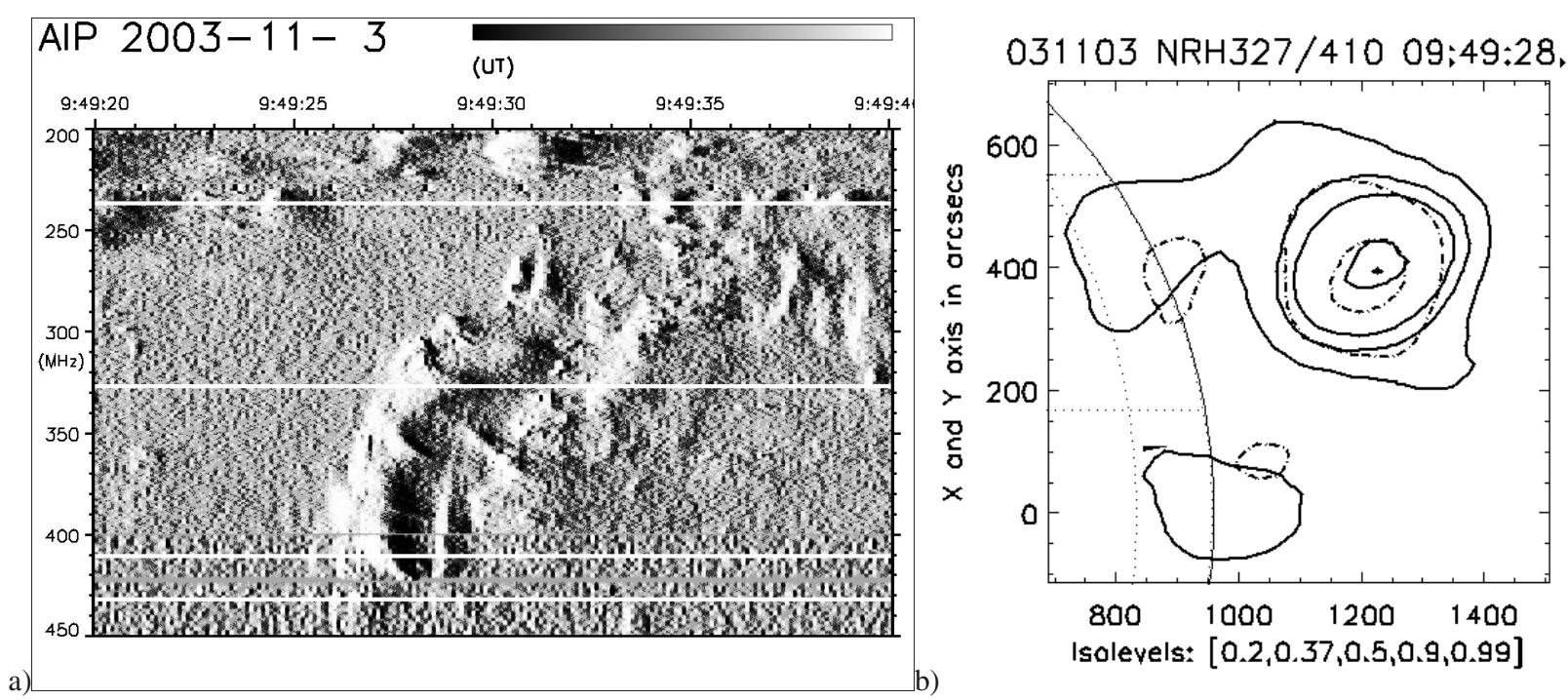

Fig. 8. Interval (2) of Fig. 2. a) The dynamic spectrum. White lines denote the NRH frequencies. b) 327 (continuous contour) and $410.5 \mathrm{MHz}$ (dash-dot contour) at 09:49:28.8 UT (all normalized as before). The upper source flux dominates, all frequencies coincide, all source sites are slightly displaced with the higher frequency (density) nearer to the flare site. The $432 \mathrm{MHz}$ source flux is below the $20 \%$ level.

ignition of fast reconnection in the CSs. The electrons, thereby energized, become radio-visible where hot, inhomogeneous, and streaming plasma is situated (in reconnection jets next to magnetic Y- and X-points as predicted by Holman 2012).

The narrowband short pulses of radio emission coming simultaneously from two widely spaced regions in the corona indicate a common electron supply for both source sites. The source spectrum itself may be harmonically structured (see Fig. 3b), with a characteristic $30 \mathrm{MHz}$ spacing toward higher frequencies. This points to a magnetic field strength of about 10 Gauss at the source site of these short and narrowband bursts of radio emission. If we consider the complex spectrum to be due to a hot coronal plasma kernel (which can never be resolved by any imaging observation), we take it as an additional reason to ask if this spectral pattern signifies the transition to anomalous resistivity in both the flare and the breakout CS.

The bright and wide bandwidth radio emission at this time was probably the result of the magnetic flux rope produced by the flare reconnection now being fully formed and suddenly exerting a force on the overlying magnetic field. The appearance of these upper sources within one second indicates rapid evolution and a sub-second electron acceleration time. The outward shift of the northeastern $236 \mathrm{MHz}$ source within just $2 \mathrm{~s}$ (Fig. 6b) also indicates rapid evolution.

The high polarization indicates that the emitting region is compact, encompassing just a fraction of the volume of the flux rope or from within the flare current sheet. Therefore, if this interpretation is correct, the electrons must only emit the pulsations as they pass through the jet region (where they will get depolarized) after traversing the length of the flux rope.

Although rapid breakout reconnection is apparently driven at 09:49:05 UT and for at least another 150 s (until the type II burst onset it remains radio-visible), the associated radio sources do not move radially outward, and we do not see the escape of a radio-emitting flux rope even though the HXR flare loop top rises freely after its height minimum passage (Fig. 2b).

It is generally accepted that radio emission as studied here is due to plasma waves excited by accelerated electrons. Assuming emission at the electron plasma frequency, or possibly the second harmonic of the plasma frequency, the frequency of

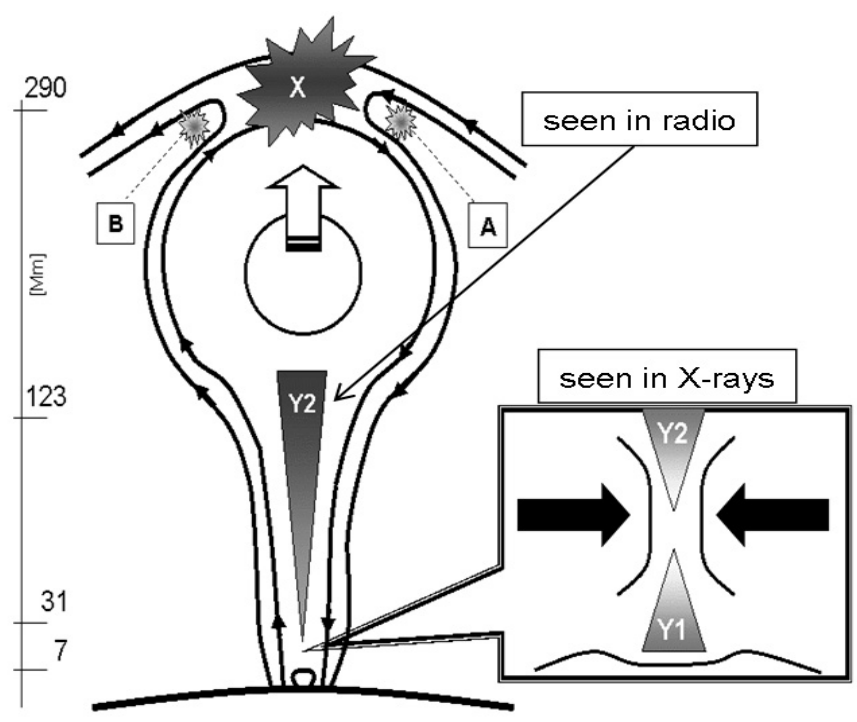

Fig. 9. Similar to Fig. 1, but with the radio and HXR sources indicated. The upper outflow jet (Y2) can be detected in HXR and radio emission. The only in radio emission visible breakout sources $\mathrm{X}, \mathrm{A}$, and $\mathrm{B}$ do not move radially during the analyzed time interval of the event.

emission is proportional to the square root of the local plasma density. Therefore, emission at $432 \mathrm{MHz}$ implies a density of $2.3 \times 10^{9} \mathrm{~cm}^{-3}$ and emission at $236 \mathrm{MHz}$ implies a density of $6.9 \times 10^{8} \mathrm{~cm}^{-3}$ (or four times smaller if the emission is at the second harmonic). It may be argued that these densities are high for an altitude of $290 \mathrm{Mm}$ in the solar atmosphere. However, this is a compressed region and coronal densities several orders of magnitude greater than this have been inferred in flaring regions at altitudes somewhat lower than this.

The emission at 09:49:05 UT (pattern (1) in Fig. 2b) is followed by a complex outburst starting at about 09:49:26 UT. After the onset of this strong and complex radio emission pattern (pattern (2) in Fig. 2b) the HXR flux rises toward its main maximum. The radio source is dominantly and at least over the frequency range $410-327 \mathrm{MHz}$ cospatially emitted 
from the breakout reconnection source site only. This demonstrates that the breakout reconnection process is most efficient at least for the radio-visible part of the spectrum of nonthermal electrons.

Consequently, we have to ask for the part of energy release which has to be ascribed to the breakout reconnection process. It depends not only on the rise speed and the magnetic field strength of the erupting structure (and so, in other words, on the properties of the flaring active region and the amount of previously stored energy), but also on the structure and the strength of the overlying large-scale magnetic flux systems.

Burst pattern (2) in Fig. 2 can be understood if the atmosphere relaxes after the attempted break out at 09:49:05 UT. As shown in Fig. 8b, the emission is from a region just northeast of the original upper $410 \mathrm{MHz}$ source with weak emission from the northern and southern flanks of the active region. Relaxation of the atmosphere means that the plasma density and magnetic field strength decrease somewhat in the region of breakout reconnection and along the expelled newly reconnected magnetic field lines. Both the shifted centroid of the $410 \mathrm{MHz}$ source and the absence of emission at $432 \mathrm{MHz}$ reflect this decrease in plasma density. The near coincidence of the 410 and $327 \mathrm{MHz}$ centroids indicates a sharp density gradient away from the location of breakout reconnection at this time. The low weaker sources (compare Fig. 8b) are emitted where the plasma density again increases downward to a value with a plasma frequency corresponding to the observational frequency. These lower sources may be visible at this time because the relaxation of the magnetic field lowers the mirror point, allowing the electrons accelerated in the breakout reconnection to reach a density and, therefore, plasma frequency that is high enough to yield observable emission. The lower frequency sources are shifted outward from the location of the higher frequency sources. This can be understood since, as the density decreases in the breakout reconnection region, the density in the newly formed outer loops will be lower than the density in the previously formed inner loops. The density in the breakout reconnection region partially recovers from about 09:49:36 UT to 09:49:44 UT. This increase in plasma density produces the tendency toward higher frequencies in the dynamic spectrum in Fig. 2a after 09:49:35 UT, this means in the seconds after feature (2).

If the nonthermal particles that are energized at the breakout reconnection site have access to the denser atmosphere in the flaring active region (which is fully expected) our result opens an additional source of flare energy which varies from event to event depending on the field orientation between flare loops and overlying magnetic flux systems. The breakout reconnection may also open a wide angular injection range of solar energetic particles away from the Sun due to the magnetic field structure alone, and without the need of azimuthal diffusion processes.
Our scenario provides a comprehensive interpretation of the different effects observed during the impulsive phase of the 2003 November 03 SEE in metric radio and hard X-ray emission. This interpretation must, of course, be tested against observations of other eruptive events. The results of this paper indicate the unique value of radio observations in the meter wave range for understanding both HXR and radio emission during SEEs, and for studying the properties and evolution of electron acceleration in both breakout and flare reconnection jets.

Acknowledgements. The authors acknowledge the use of radio imaging data of the French Multi-Frequency Radioheliograph in Nançay, and the open data policy of the team running this instrument. H.A. thanks A. Kerdraon for advice concerning the NRH data. We are also obliged to A. Veronig and S. Krucker (RHESSI data), H. Meszarosova, M. Karlický (Astronomical Observatory Ondřejov, Czech Republic), and W. Pötzi (Univ. Graz, Obs. Kanzelhöhe, Austria) for information about observations. We took profit from the use of SOHO and GOES-SXI data. The authors gratefully acknowledge the helpful comments of an unknown referee. G.D.H. acknowledges support from the RHESSI Project and a NASA Guest Investigator Grant. This work was stimulated by the participation of H.A. and S.B. in the EU FP7-SPACE2010-1program under grant no. 262773. The work of S.B. was also supported by the DLR grant No. 50QL0901.

\section{References}

Altyntsev, A. A., Fleishman, G. D., Lesovoi, S. V., \& Meshalkina, N. S. 2012, ApJ, 758, 138

Antiochos, S. K., DeVore, C. R., \& Klimchuk, J. A. 1999, ApJ, 510, 485

Aurass, H., \& Klein, K.-L. 1997, A\&AS, 123, 279

Aurass, H., Mann, G., Zlobec, P., \& Karlický, M. 2011, ApJ, 730, 57

Bastian, T. S., Benz, A. O., \& Gary, D. E. 1998, ARA\&A, 36, 131

Chen, B., Bastian, T. S., Gary, D. E., \& Jing, J. 2011, ApJ, 736, 64

Chen, Q., \& Petrosian, V. 2012, ApJ, 748, 33

Dauphin, C., Vilmer, N., Lüthi, T., et al. 2005, Adv. Space Res., 35, 1805

Dauphin, C., Vilmer, N., \& Krucker, S. 2006, A\&A, 455, 339

Holman, G. D. 2012, Physics Today, 04, 56

Holman, G. D., Aschwanden, M. J., Aurass, H., et al. 2011, Space Sci. Rev., 159, 107

Karpen, J. T., Antiochos, S. K., \& DeVore, C. R. 2012, ApJ, 760, 81

Lin, J., \& Forbes, T. G. 2000, J. Geophys. Res., 105, 2375

Lin, J., Raymond, J. C., \& van Ballegooijen, A. A. 2004, ApJ, 602, 422

Liu, W., Petrosian, V., Dennis, B. R., \& Holman, G. D. 2009, ApJ, 693, 847

MacNeice, P., Antiochos, S. K., Phillips, A., et al. 2004, ApJ, 614, 1028

Mann, G., \& Klassen, A. 2005, A\&A, 441, 319

Mann, G., \& Warmuth, A. 2011, A\&A, 528, A104

Melrose, D. B. 1985, in Solar Radiophysics, eds. D. J. McLean, \& N. R. Labrum (Cambridge Univ. Press)

Priest, E., \& Forbes, T. 2000, Magnetic Reconnection (Cambridge Univ. Press)

Somov, B. 2000, Cosmic Plasma Physics (Dordrecht: Kluwer)

Spicer, D. S. 1977, Sol. Phys., 53, 305, 1977

Sui, L., Holman, G. D., \& Dennis, B. R. 2004, ApJ, 612, 546

Svestka, Z. 1976, Solar Flares, Geophys. Astrophys. Monographs (Dordrecht: Reidel), 8

Veronig, A. M., Karlický, M., Vršnak, B., et al. 2006, A\&A, 446, 675

Vršnak, B., Magdalenić, J., Temmer, M., et al. 2005, ApJ, 625, L67

Vršnak, B., Warmuth, A., Temmer, M., et al. 2006, A\&A, 448, 739

Zlotnik, E. Ya., Zaitsev, V. V., Aurass, H., Mann, G., \& Hofmann, A. 2003, A\&A 410, 1011 